\title{
Branch Duct
}

National Cancer Institute

\section{Source}

National Cancer Institute. Branch Duct. NCI Thesaurus. Code C113668.

A small duct that originates from the main duct. 